\title{
Sarcoidosis: rarely a single system disorder
}

Sarcoidosis is a systemic disease with a number of extrapulmonary manifestations, rarely limited to a single system. The objective of this review is to provide a overview of the systemic features of sarcoidosis and their surveillance.

Sarcoidosis is disease that can affect any organ system, resulting in a number of complications that can cause significant morbidity and mortality, including ophthalmological and cardiac complications. A number of investigations play a role in identifying manifestations of sarcoidosis. Respiratory physicians have an important role to play in identifying complications and monitoring patients with sarcoidosis, as they may be the first to encounter them.

\section{Introduction}

Traditionally considered a respiratory or rheumatological disease, sarcoidosis can affect any number of organ systems with varying degrees of severity. Sarcoidosis most typically presents in patients aged $20-60$ years and the prevalence varies between ethnic groups with a high incidence in northern European countries (5-40 cases per 100000 [1]). Given the range of clinical features (table 1), respiratory physicians should be aware of the possible extrapulmonary manifestations of sarcoidosis, as they may be the first to encounter them.

This review will discuss the extrapulmonary manifestations and complications of sarcoidosis which have been discussed in more detail elsewhere $[2,3]$.

\section{Ophthalmological}

Ocular involvement, often bilateral, after respiratory and rheumatological diseases, is the third most common organ manifestation of sarcoidosis [3]. The typical presentation of ocular sarcoidosis is uveitis, which can be subdivided into anterior, posterior, intermediate and diffuse dependent on the location. Anterior uveitis may present as pain, redness and vision loss, whereas posterior uveitis is usually painless visual loss with "floaters" [3, 4].

Approximately one-third of ocular sarcoidosis is not uveitis, and includes conjunctivitis, scleritis, lacrimal gland involvement, optic neuritis and conjunctival nodules [4]. With the exception of optic neuritis, these manifestations often spare the vision and respond well to treatment [5]. The conjunctivae
Cite as: Mannes K, Thomas PS. Sarcoidosis: rarely a single system disorder. Breathe 2020; 16: 200207.

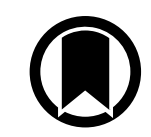

CrossMark

C ERS 2020 
Table 1 Extrapulmonary manifestations of sarcoidosis and appropriate investigations

\begin{tabular}{|c|c|c|c|}
\hline Organ system & Prevalence & Manifestations & Investigations \\
\hline Systemic & & Fatigue, malaise, weight loss, fever & Serum inflammatory markers, serum ACE \\
\hline Ocular & $20-50 \%$ & Uveitis, conjunctivitis, nodules, optic neuritis & Ophthalmological assessment, Gallium scan (rare \\
\hline Cardiac & $5-25 \%$ & $\begin{array}{l}\text { Heart block, dysrhythmias, heart failure, } \\
\text { valvular dysfunction, pericardial disease, } \\
\text { sudden death }\end{array}$ & $\begin{array}{l}\text { Electrocardiography, echocardiography, Holter } \\
\text { monitoring, cardiac MRI }\end{array}$ \\
\hline $\begin{array}{l}\text { Renal and } \\
\text { endocrine }\end{array}$ & $2-10 \%$ & $\begin{array}{l}\text { Hypercalcaemia and hypercalciuria, } \\
\text { interstitial nephritis, nephrolithiasis, } \\
\text { nephrocalcinosis, hormonal dysfunction }\end{array}$ & $\begin{array}{l}\text { Renal function testing (serum electrolytes, urea, } \\
\text { creatinine), serum calcium, serum vitamin D, } \\
\text { ultrasonography, hormone studies }\end{array}$ \\
\hline $\begin{array}{l}\text { Cutaneous/ } \\
\text { lymphoid }\end{array}$ & $25 \%$ & $\begin{array}{l}\text { Erythema nodosum, lupus pernio, } \\
\text { nonspecific patterns (e.g. papular, nodular, } \\
\text { plaque-like), palpable lymphadenopathy }\end{array}$ & $\begin{array}{l}\text { Biopsy as needed, skin lesions can often be } \\
\text { clinically diagnosed }\end{array}$ \\
\hline Musculoskeletal & $25-39 \%$ & Arthropathy, myopathy, bony lesions & $\begin{array}{l}\text { Serum creatinine kinase, radiographs, } \mathrm{CT}, \mathrm{MRI} \text {, } \\
\mathrm{PET} \text {, muscle biopsy }\end{array}$ \\
\hline $\begin{array}{l}\text { Nervous } \\
\text { system }\end{array}$ & $10 \%$ & $\begin{array}{l}\text { Headaches, cranial nerve palsies, cognitive } \\
\text { and psychiatric dysfunction, small fibre } \\
\text { neuropathy, seizures, spinal neuropathy }\end{array}$ & $\begin{array}{l}\text { Brain and spine MRI, CSF analysis, } \\
\text { electromyography, nerve conduction studies }\end{array}$ \\
\hline $\begin{array}{l}\text { Liver and } \\
\text { spleen }\end{array}$ & $30-80 \%$ & $\begin{array}{l}\text { Hepatomegaly, splenomegaly, nonspecific } \\
\text { liver function and enzyme derangement }\end{array}$ & $\begin{array}{l}\text { Liver function testing, abdominal ultrasound } \\
\text { and } \mathrm{CT} \text {, liver biopsy if significant laboratory } \\
\text { derangement }\end{array}$ \\
\hline Haematological & $4-40 \%$ & Anaemia, leukopenia & $\begin{array}{l}\text { Full blood count with differential, bone marrow } \\
\text { biopsy (rare) }\end{array}$ \\
\hline
\end{tabular}

Pulmonary manifestations are not included. ACE: angiotensin converting enzyme; CT: computed tomography; MRI: magnetic resonance imaging; PET: positron emission tomography; CSF: cerebrospinal fluid. Data from [3, 4, 8, 38, 44, 45].

can biopsied when involved and this avoids more invasive sampling.

All patients with sarcoidosis should have regular, probably annual, ophthalmological review regardless of the presence of symptoms, and patients should be asked about symptoms whenever being reviewed by respiratory physicians.

\section{Cardiac}

Although rare, cardiac involvement of sarcoidosis is crucial to recognise, as it has the potential to be life-threatening. After respiratory failure, it is the second-most common cause of sarcoidosisrelated mortality in most countries, and in Japan it is the predominant cause $[6,7]$. The prevalence across ethnic groups varies, and the diagnostic criteria are imprecise, however it appears to be approximately $5 \%$ but is limited by ascertainment bias in the studies [7, 8]. There is a predilection for the left ventricular wall, interventricular septum and conducting system, but any part of the heart can be affected [9].

Patients with cardiac involvement may be asymptomatic, or present with chest pain, palpitations, dyspnoea, congestive heart failure, pericardial effusion or syncope/presyncope due to dysrhythmias. The most common abnormality is atrioventricular block [10]. For this reason, an ECG with any conduction defect (bundle branch block, prolonged PR interval) or other nonspecific changes should prompt further cardiological studies.

Echocardiograms have a low sensitivity and, realistically, more advanced imaging, such as cardiac magnetic resonance imaging (MRI) or ${ }^{18} \mathrm{~F}$-fluorodeoxyglucose cardiac positron emission tomography (PET) is more sensitive, though more costly [11]. There is a lack of evidence as to whether the initial investigation should be cardiac MRI or cardiac PET, but it seems likely that the former is able to detect areas of fibrosis while the latter detects active inflammatory disease. It may be that cardiac MRI will be a useful baseline modality, but CPET will be more useful to guide anti-inflammatory treatment decisions.

Endomyocardial biopsy has a low ( $30 \%)$ sensitivity [12] given the patchy nature of cardiac sarcoidosis and is an invasive procedure. There are various proposed diagnostic criteria for cardiac sarcoidosis, for instance, the Heart Rhythm Society [13] provides two pathways for diagnosis: histological diagnosis from myocardial tissue, and "probable" diagnosis using histological extracardiac evidence, exclusion of other causes, plus other minor criteria including PET uptake, steroid responsiveness, heart block or unexplained reduced 
left ventricular ejection fraction [13]. Similar criteria exist elsewhere, such as the Japanese Circulation Society guidelines, which utilise major and minor criteria $[14,15]$.

A further controversial issue in cardiac sarcoidosis is when to use an implantable cardioverter defibrillator and risk stratification for sudden death in sarcoidosis patients. There is a distinct lack of evidence to guide implantable cardioverter defibrillator use, although placement can, of course, be lifesaving [6].

A reasonable approach in all patients is a baseline ECG, with symptoms or abnormal ECG findings warranting further investigation including echocardiography, 24-h Holter monitoring and consideration of cardiac MRI or cardiac PET, but this advice is not based on clinical trials.

\section{Renal and endocrine}

Renal sarcoidosis is classically a granulomatous interstitial nephritis; however, clinically significant nephritis is rare (around 3\%) [4]. The majority of patients with renal involvement have evidence of sarcoidosis in other organs and disease is rarely limited to the kidneys alone [16]. Renal biopsy is often used as a diagnostic tool; however, the results are often nonspecific, perhaps in part due to the patchy nature of the sarcoidosis infiltrate. Aside from granulomatous interstitial nephritis, membranous nephropathy, diffuse proliferative or crescentic glomerulonephritis and focal segmental glomerulosclerosis have all been described in biopsy of suspected cases of renal sarcoidosis [17].

Hypercalcaemia occurs in approximately $10-20 \%$ of sarcoidosis patients as a result of excess hydroxylated vitamin $D$ created by macrophages within sarcoid granulomas [18]. This predisposes the patient to hypercalciuria, which in turn can lead to nephrolithiasis and nephrocalcinosis. Monitoring serum calcium, renal function (serum electrolytes, urea, creatinine) and an initial 24-h urinary calcium would be appropriate [3]. The American Thoracic Society statement suggests that if vitamin $D$ replacement is indicated, measuring both 25 - and $1,25-\mathrm{OH}$ vitamin $D$ levels before vitamin $D$ replacement would be appropriate, but the interpretation of the results can be difficult [2].

Serum angiotensin converting enzyme (ACE), whilst nonspecific and only modestly sensitive, is elevated in $60-80 \%$ of patients with sarcoidosis, and may reflect total granuloma burden [3].

Pituitary gland and hypothalamic involvement can result in impaired hormone secretion, diabetes insipidus, and syndrome of inappropriate antidiuretic hormone secretion [3]. Rarely, the adrenal glands may be affected, causing adrenal insufficiency.

\section{Dermatological}

Cutaneous involvement is present in up to one-third of those with sarcoidosis and varies significantly between patients [4].

The most common nonspecific sarcoidosis skin lesion is erythema nodosum [4], which is found in $10 \%$ of patients [3]. Biopsy of erythema nodosum is not useful for diagnosis as it often displays nonspecific panniculitis [3]. It can often indicate an acute form of sarcoidosis and is found in concert with arthritis and bilateral hilar adenopathy (Löfgren's syndrome) [19]. Löfgren's syndrome spontaneously resolves within 6 months in most patients [19, 20]. Therefore, patients with erythema nodosum need a chest radiograph and other tests to look for other causes of the presentation (e.g. primary tuberculosis and other infections, drug reactions, inflammatory bowel disease, etc.).

The most common "sarcoidosis-specific" skin diseases (i.e. evidence of granulomas on biopsy) are subcutaneous nodules and papules/plaques [19]. These are frequently found on the extremities and head and neck and vary markedly in appearance, often multiple and usually painless.

Lupus pernio is also a sarcoidosis-specific skin lesion which appears as erythematous to violet papules/plaques on the face, in particular the alar rim [21]. Patients may also experience inflammation around scars or tattoos, the Köbner phenomenon [22].

Biopsy of sarcoidosis-specific cutaneous lesions can be an option to demonstrate noncaseating sarcoidal granulomas if histopathological confirmation has not already been obtained, avoiding more invasive methods, and with a sensitivity of up to $90 \%$ [19].

\section{Musculoskeletal}

Sarcoidosis can be found in all parts of the musculoskeletal system. Arthropathy is found in a significant percentage of patients (5-15\%) and the most typical presentation is that of acute oligoarthritis in the ankle joint (>90\% of cases) [23]. Unlike rheumatoid or osteoarthritis, polyarthritis of the small hand joints is uncommon, although it can extend to the knees, wrists and elbows [24]. Close liaison with rheumatologists will aid the management, and also provide a link for their sarcoidosis patients who should likewise have regular pulmonary review.

In terms of muscular involvement, sarcoid myopathy can be acute, chronic or nodular, with chronic being most common [3]. Classically this presents as symmetrical, proximal weakness and can be difficult to diagnose, with muscle biopsy being helpful when diagnosis is unclear [25]. Bony involvement is usually asymptomatic and hence an incidental finding on imaging. 
The most common related complaint is of back pain with those who have vertebral involvement [26]. Histologically, non-caseating granulomas are most often found in the synovium and musculature, whilst lytic and destructive lesions are found in bone [18]. Usually these lesions do not require treatment, but can be mistaken for malignant lytic lesions [24, 27].

\section{Neurological}

Neurosarcoidosis is uncommon, but can be a devastating complication when manifesting in the central nervous system. It manifests in 10\% of patients with multisystem sarcoidosis [28] and commonly presents as cranial nerve palsies, headaches, weakness, seizures and neuropsychiatric complaints. Seizures are associated with chronicity and increased morbidity [29].

Neurosarcoidosis and sarcoidosis-associated myelopathy [30] can prove difficult to diagnose definitively given the potential for neurological damage if biopsied. The diagnosis may be based on the clinical picture in conjunction with extraneural organ involvement or suggestive MRI findings (including but not limited to leptomeningeal enhancement, dural enhancement, and intraparenchymal lesions [31]) or cerebrospinal fluid analysis (monocyte pleocytosis and a high protein level) [4]. The diagnosis is usually clinical, and while the cerebrospinal fluid ACE level has a poor sensitivity and specificity, it is often used in the absence of better indicators [32]. PET or gallium-67 scan can sometimes help to detect disease in other tissues that may be amenable to biopsy.

\section{Hepatic and splenic}

The reported prevalence of hepatic sarcoidosis varies greatly, from $5 \%$ to $30 \%$ [33], but may be as high as $80 \%$ in post mortem autopsy studies [34]. Hepatic sarcoidosis is usually asymptomatic, and discovered incidentally by abnormal liver biochemistry, haematological abnormalities or abdominal imaging [4]

The most common pattern of abnormality is an elevated alkaline phosphatase and $\gamma$-glutamyltransferase, a reflection of sarcoid infiltration [4]. These are typically more than three times the upper limit of normal. Alanine aminotransferase and aspartate aminotransferase are less often elevated, and when they are, it is often to a lesser extent [33].

Liver biopsy has largely been replaced by various imaging modalities including ultrasonic transient elastography ("fibroscan"), computed tomography (CT) and MRI, but biopsy remains the only definitive test; particularly if there is doubt as to the cause of hepatic dysfunction [3]. Imaging will often show hypodense lesions in the liver and spleen, as well as hepatosplenomegaly. Splenomegaly is not uncommon, but usually minimal, although can on occasion lead to pressure symptoms [3].

A minority of patients will proceed to cirrhosis of the liver (6-24\%); however, overall the prognosis of hepatic sarcoidosis is good, with many patients having spontaneous resolution of biochemical abnormalities [33].

\section{Haematological}

Anaemia is a remarkably common occurrence in those with sarcoidosis, present in $4-20 \%$ of

Table 2 Suggestions for ongoing monitoring of patients diagnosed with sarcoidosis, based on expert opinion $\#$

\begin{tabular}{|c|c|}
\hline Investigation 9 & Frequency $^{+}$ \\
\hline $\begin{array}{l}\text { Blood tests including full blood count, electrolytes, urea, } \\
\text { creatinine, metabolic panel (serum calcium, magnesium, } \\
\text { phosphate), liver function tests, ACE level }\end{array}$ & $\begin{array}{l}\text { Initial diagnosis then quarterly until stable, or monthly if on } \\
\text { immunosuppression }\end{array}$ \\
\hline Electrocardiogram & Initial diagnosis then at least biannual \\
\hline Ophthalmological review & Annual \\
\hline Chest radiograph & Initial diagnosis \\
\hline Chest CT & $\begin{array}{l}\text { Depending on progress, but perhaps at least biannual if } \\
\text { progressive disease }\end{array}$ \\
\hline \multirow[t]{2}{*}{ Urinalysis } & Initial diagnosis, with 24 h urinary calcium \\
\hline & Annual \\
\hline \multirow[t]{3}{*}{ Follow-up } & Quarterly in active disease \\
\hline & Every $12-18$ months in inactive disease for $>2$ years \\
\hline & Some recommend at least 5 year follow-up \\
\hline
\end{tabular}

\#: prospective data is lacking; १: further investigation is warranted if abnormalities are found or dependent on symptoms; ${ }^{+}$: frequency may be increased dependent on patient symptoms and known organ involvement. 
patients [35]. Leukopenia too is found in up to $40 \%$ of patients, but is most often mild. Lymphocytopenia is common and probably due to the accumulation of activated T-cells in granulomatous sites (hence removing them from peripheral circulation) [36]. Rarely, patients may also have eosinophilia, thrombocytopenia or even autoimmune haemolytic anaemia [37].

One-third of sarcoidosis patients will have palpable peripheral lymphadenopathy, typically cervical, inguinal and axillary. They are mobile and non-tender [3], and useful for histopathological diagnosis.

\section{Gastrointestinal}

Technically, any part of the gastrointestinal tract may also be affected by sarcoidosis; however, the overall prevalence appears to be $<1 \%$ [38, 39]. Those affected most commonly present with epigastric pain if symptomatic. Other presentations may include nausea, early satiety and weight loss, with obstructive symptoms arising due to external compression of the gut lumen by lymphadenopathy [3].

The pancreas may also be infiltrated by sarcoidosis, diffusely or in the pancreatic head, which may result in an obstructive picture and/or lipase elevation [18] mimicking a neoplasm.

\section{Systemic and psychosocial}

There are a number of other nonspecific complaints in sarcoidosis; including fever, weight loss, anorexia, night sweats, sleep changes, lethargy and malaise. Fever tends to be low grade but can reach up to $40^{\circ} \mathrm{C}$ [3]. Other diseases need to be excluded for these nonspecific complaints.

It is also important to recognise the psychosocial impacts of sarcoidosis on patients and families. Depression is particularly common, with prevalence ranging up to $66 \%$ [40], and one study showing

\section{Self-evaluation questions}

1. Which of the following statements is correct?

a) Skin involvement is found in every patient with sarcoidosis

b) Renal biopsy is often used for diagnosis as it usually shows specific histopathological evidence of granulomas

c) Serum ACE is a sensitive test for screening for sarcoidosis

d) Biopsy of heart muscle will always show histopathological evidence of sarcoidosis if affected

e) Every patient with sarcoidosis should have a baseline electrocardiogram, as cardiac involvement may be asymptomatic

2. True or false:

a) Ophthalmological review of patients with sarcoidosis is only indicated in symptomatic patients

b) Erythema nodosum is a sarcoidosis-specific skin finding

c) All patients with sarcoidosis should have a baseline ECG

d) Gastrointestinal involvement is a common manifestation of sarcoidosis and a major cause of morbidity

prevalence of anxiety at 32\% [41]. Anecdotally, websites can provoke anxiety in many patients who read of those afflicted by life-threatening sarcoidosis, and often require reassurance. Fatigue alone can persist even in remission of sarcoidosis, having significant effects on quality of life in addition to being associated with low mood $[42,43]$.

\section{Summary}

Sarcoidosis is rarely a single system disease and has an array of possible manifestations. As a respiratory physician, to focus on pulmonary manifestations alone risks under-recognising the significant complications of sarcoidosis, in turn missing significant morbidity and even potential mortality. There remains a need for high-quality evidence to guide clinical practice in diagnosing and monitoring sarcoidosis.

\section{Key points}

- Sarcoidosis is a systemic disease which can affect any organ system

- Respiratory physicians play an important role in recognising extrapulmonary complications of sarcoidosis

- If a patient with known pulmonary sarcoidosis presents with extrapulmonary organ dysfunction, sarcoidosis may be suspected as the causative process

- A table of recommendations for ongoing investigations is included (table 2); this is based on expert opinion as prospective data is lacking 


\section{Affiliations}

\section{Katrin Mannes ${ }^{1,2}$, Paul S. Thomas ${ }^{1,2,3}$}

'Dept of Respiratory Medicine, Prince of Wales' Hospital, Sydney, Australia. 2Prince of Wales' Clinical School, Faculty of Medicine, University of New South Wales, Sydney, Australia. ${ }^{3}$ Mechanisms of Disease and Translational Research, Faculty of Medicine, University of New South Wales, Sydney, Australia.

\section{Acknowledgements}

P.S. Thomas would like to acknowledge two of his mentors for his interest in sarcoidosis, D.G. James (Royal Northern and Royal Free Hospitals, London, UK) and D.N. Mitchell (Royal Brompton Hospital, London, UK).

\section{Conflict of interest}

K. Mannes has nothing to disclose. P.S. Thomas has nothing to disclose.

\section{Suggested}

\section{answers}

1. e

2. a) false, b) false, c) true, d) false

\section{References}

1. Pietinalho A, Hiraga $Y$, Hosoda $Y$, et al. The frequency of sarcoidosis in Finland and Hokkaido, Japan. A comparative epidemiological study. Sarcoidosis 1995; 12: 61-67.

2. Crouser E, Maier L, Wilson K, et al. Diagnosis and Detection of Sarcoidosis. An Official American Thoracic Society Clinica Practice Guideline. Am J Respir Crit Care Med 2020; 201: e26-e51.

3. Ahmadzai H, Huang S, Steinfort C, et al. Sarcoidosis: a state of the art review from the Thoracic Society of Australia and New Zealand. Med J Aust 2018; 208: 499-504.

4. Ungprasert P, Ryu J, Matteson E. Clinical manifestations, diagnosis, and treatment of sarcoidosis. Mayo Clin Proc 2019; 3: 358-375.

5. Rothova A, Groen F. Ocular involvement in sarcoidosis. Semin Respir Crit Care Med 2017; 38: 514-522.

6. Sauer W, Stern B, Baughman R, et al. High-risk sarcoidosis. Current concepts and research imperatives. Ann Am Thorac Soc 2017; 14: Suppl. 6, S437-S444.

7. Morimoto T, Azuma A, Abe S, et al. Epidemiology of sarcoidosis in Japan. Eur Respir J 2008; 31: 372-379.

8. Baughman RP, Teirstein AS, Judson MA, et al. Clinical characteristics of patients in a case control study of sarcoidosis. Am J Respir Crit Care Med 2001; 164: 1885-1889.

9. Nunes H, Freynet O, Naggara N, et al. Cardiac sarcoidosis. Semin Respir Crit Care Med 2010; 31: 428-441.

10. Kandolin R, Lehtonen J, Airaksinen J, et al. Cardiac sarcoidosis: epidemiology, characteristics, and outcome over 25 years in a nationwide study. Circulation 2015; 131: 624-632.

11. Schatka I, Bengel F. Advanced imaging of cardiac sarcoidosis. J Nucl Med 2014; 55: 99-106.

12. Ardehali H, Howard DL, Hariri A, et al. A positive endomyocardial biopsy result for sarcoid is associated with poor prognosis in patients with initially unexplained cardiomyopathy. Am Heart J 2005; 150: 459-463.

13. Birnie DH, Sauer WH, Bogun F, et al. HRS expert consensus statement on the diagnosis and management of arrhythmias associated with cardiac sarcoidosis. Heart Rhythm 2014; 11 1305-1323.

14. The Japanese Circulation Society (JCS). Guidelines for the diagnosis and treatment of cardiac sarcoidosis (JCS 2016) [in Japanese]. www.j-circ.or.jp/guideline/pdf/JCS2016_ terasaki_h.pdf Date last updated: 24 February 2017.

15. Terasaki F, Yoshinaga K. New guidelines for diagnosis of cardiac sarcoidosis in Japan. Ann Nucl Cardiol 2017; 3: 42-45.

16. Kamata Y, Sato H, Joh K, et al. Clinical characteristics of biopsyproven renal sarcoidosis in Japan. Sarcoidosis Vasc Diffuse Lung Dis 2018; 35: 252-260

17. Shah R, Shidham G, Agarwal A, et al. Diagnostic utility of kidney biopsy in patients with sarcoidosis and acute kidney injury. Int J Nephrol Renovasc Dis 2011; 4: 131-136.
18. Rao DA, Dellaripa PF. Extrapulmonary manifestations of sarcoidosis. Rheum Dis Clin North Am 2013; 39: 277-297.

19. Ungprasert P, Wetter DA, Crowson CS, et al. Epidemiology of cutaneous sarcoidosis, 1976-2013: a population-based study from Olmsted County, Minnesota. J Eur Acad Dermatol Venereol 2016; 30: 1799-1804.

20. Mañá J, Gómez-Vaquero C, Montero A, et al. Löfgren's syndrome revisited: a study of 186 patients. Am J Med 1999; 107: 240-245.

21. Katta R. Cutaneous sarcoidosis: a dermatologic masquerader. Am Fam Physician 2002; 65: 1581-1585.

22. Marchell RM, Judson MA. Cutaneous sarcoidosis. Semin Respir Crit Care Med 2010; 31: 442-451.

23. Ungprasert P, Crowson C, Matteson E. Clinical characteristics of sarcoid arthropathy: a population-based study. Arthritis Care Res (Hoboken) 2016; 68: 695-699.

24. Bechman K, Christidis D, Walsh S, et al. A review of the musculoskeletal manifestations of sarcoidosis. Rheumatology 2017; 57: 777-783.

25. Yanardag H, Tetikkurt C, Bilir M. Clinical and prognostic significance of muscle biopsy in sarcoidosis. Monaldi Arch Chest Dis 2018; 88: 910.

26. Lisle D, Mitchell K, Crouch M, et al. Sarcoidosis of the thoracic and lumbar spine: imaging findings with an emphasis on magnetic resonance imaging. Australas Radiol 2004; 48: 404-407.

27. Sparks JA, McSparron JI, Shah N, et al. Osseous sarcoidosis: clinical characteristics, treatment, and outcomes - experience from a large, academic hospital. Semin Arthritis Rheum 2014; 44: 37-39.

28. Joseph FG, Scolding NJ. Neurosarcoidosis: a study of 30 new cases. J Neurol Neurosurg Psychiatry 2009; 80: 297-304

29. Sharma OP. Cardiac and neurologic dysfunction in sarcoidosis. Clin Chest Med 1997; 18: 813-825.

30. Murphy O, Salazar-Camelo A, Jimenez J, et al. Clinical and MR phenotypes of sarcoidosis-associated myelopathy. Neurology 2020; 7: e722.

31. Ginat D, Dhillon G, Almast J. Magnetic resonance imaging of neurosarcoidosis. J Clin Imaging Sci 2011; 1: 15.

32. Khoury J, Wellik KE, Demaerschalk BM, et al. Cerebrospinal fluid angiotensin-converting enzyme for diagnosis of central nervous system sarcoidosis. Neurologist 2009; 15: 108-111.

33. Ungprasert $P$, Crowson CS, Simonetto DA, et al. Clinical characteristics and outcome of hepatic sarcoidosis: a population-based study 1976-2013. Am J Gastroenterol 2017; 112: 1556-1563.

34. Ricker W, Clark M. Sarcoidosis: a clinicopathologic review of three hundred cases, including twenty-two autopsies. Am J Clin Pathol 1949; 19: 725-749.

35. Lower EE, Smith JT, Martelo OJ, et al. The anemia of sarcoidosis. Sarcoidosis 1988; 5: 51-55. 
36. Gerke AK, Hunninghake $G$. The immunology of sarcoidosis. Clin Chest Med 2008; 29: 379-390.

37. Nunes $\mathrm{H}$, Soler P, Valeyre D. Pulmonary sarcoidosis. Allergy 2005; 60: 565-582.

38. Ungprasert P, Carmona E, Utz J, et al. Epidemiology of sarcoidosis 1946-2013. Mayo Clin Proc 2016; 91 183-188.

39. Afshar K, Boyd King A, Sharma OP, et al. Gastric sarcoidosis and review of the literature. J Natl Med Assoc 2010; 102: 419-422.

40. Drent M, Wirnsberger RM, Breteler MH, et al. Quality of life and depressive symptoms in patients suffering from sarcoidosis. Sarcoidosis Vasc Diffuse Lung Dis 1998; 15: 59-66.
41. Ireland J, Wilsher M. Perceptions and beliefs in sarcoidosis. Sarcoidosis Vasc Diffuse Lung Dis 2010; 27: 36-42.

42. Korenromp IH, Heijnen CJ, Vogels OJ, et al. Characterization of chronic fatigue in patients with sarcoidosis in clinical remission. Chest 2011; 140: 441-447.

43. Cox CE, Donohue JF, Brown CD, et al. Health-related quality of life of persons with sarcoidosis. Chest 2004; 125: 997-1004.

44. Baughman RP, Culver DA, Judson MA. A concise review of pulmonary sarcoidosis. Am J Respir Crit Care Med 2011; 183: 573-581.

45. Soto-Gomez N, Peters J, Nambia A. Diagnosis and management of sarcoidosis. Am Fam Physician 2016; 93 : 840-850. 